\title{
Microbubbles as a contrast agent in grating interferometry mammography: an ex vivo proof-of-mechanism study
}

\author{
Kristina Lång ${ }^{1,2^{*}}$ (D), Carolina Arboleda ${ }^{1,2}$, Serafino Forte ${ }^{3}$, Zhentian Wang ${ }^{1,2}$, Sven Prevrhal ${ }^{4}$, Thomas Koehler ${ }^{4}$, \\ Norbert Kuhn ${ }^{4}$, Bernd David ${ }^{4}$, Konstantins Jefimovs ${ }^{1,2}$, Rahel A. Kubik-Huch ${ }^{3}$ and Marco Stampanoni ${ }^{1,2}$
}

\begin{abstract}
Grating interferometry mammography (GIM) is an experimental breast imaging method at the edge of being clinically implemented. Besides attenuation, GIM can measure the refraction and scattering of $x$-rays resulting in differential phase contrast (DPC) and dark-field (DF) images. In this exploratory study, we assessed the feasibility of using microbubbles as a contrast agent in GIM. Two millilitres of microbubbles and iodine were respectively injected into ex vivo breast phantoms, consisting of fresh chicken breasts. Native and postcontrast images were acquired with a clinically compatible GIM setup, operated at $38 \mathrm{kVp}$, 14-s acquisition time, and with a dose of $1.3 \mathrm{mGy}$. The visibility of the contrast agents was analysed in a side-by-side comparison by three radiologists. The contrast-tonoise-ratio (CNR) was calculated for each contrast agent. We found that both contrast agents were judged to be visible by the readers. The mean CNR was $3.1 \pm 1.9$ for microbubbles in DF and $24.2 \pm 6.5$ for iodine in attenuation. In conclusion, this is a first proof-of-mechanism study that microbubbles could be used as a contrast agent in clinically compatible GIM, due to their scattering properties, which implies the potential use of a contrast agent with a high safety profile in $\mathrm{x}$-ray-based breast imaging.
\end{abstract}

Keywords: Contrast media, Interferometry, Mammography, Microbubbles, Phantoms (imaging)

\section{Key points}

- Grating interferometry mammography is a novel breast imaging technique at the edge of being clinically implemented

- Grating interferometry mammography obtains attenuation, phase-contrast, and dark-field images in one acquisition

- Microbubbles scatter $x$-rays, which has yet to be demonstrated in a clinical setup

- Microbubbles were shown to give contrast enhancement in the dark-field mode

\footnotetext{
* Correspondence: kristina.lang@psi.ch

'Swiss Light Source, ETH Zurich, Paul Scherrer Institute, 5232 Villigen, Switzerland

${ }^{2}$ Institute for Biomedical Engineering, University and ETH Zurich, Zurich,

Switzerland

Full list of author information is available at the end of the article
}

- Microbubbles could potentially be used as a contrast agent in clinically compatible grating interferometry mammography

\section{Background}

In conventional $x$-ray imaging, the attenuation of $x$-rays is used to create an image. However, $x$-rays are not only attenuated by matter but further refracted and scattered. So far, these signals have not been considered in medical imaging, but have recently been the focus of the developing research field of phase-contrast imaging [1, 2]. With the advent of the $\mathrm{x}$-ray grating interferometry technique, the refraction and the small-angle and ultra-small angle scattering signals can be measured resulting in differential phase contrast (DPC) and darkfield (DF) images, in addition to the attenuation image.

The grating interferometry approach has enabled the translation of phase-contrast imaging to a clinical setting, since it works with a conventional $\mathrm{x}$-ray tube and detector [3]. This emerging technique has been of special interest 
in the breast imaging field, with several potential clinical benefits [2, 4-7]. DPC provides edge enhancement which can improve soft tissue contrast [8], and the DF signal can be used to characterise tissues based on their scattering properties. As an example of the latter, it has been shown that the DF signal has the potential to discriminate between different types of microcalcifications and could enable the opportunity to non-invasively distinguish between microcalcifications associated with benign lesions and those associated with malignant lesions, which in turn can become an important clinical tool to reduce false positives and biopsy rates [9-11]. However, these studies have been performed in a preclinical setting and further evaluation in a clinical context is required.

Furthermore, the additional imaging signals with GIM could also open up new possibilities for the use of contrast agents. In breast imaging, there are currently several modalities that gain increased diagnostic performance by adding contrast agents to visualise tumour angiogenesis, such as gadolinium-enhanced breast magnetic resonance imaging (MRI), iodine-enhanced spectral mammography, and contrast-enhanced ultrasound using microbubbles $[12,13]$. However, the safety profiles of these contrast agents differ. In recent years, concerns have been raised on the potential harm of using gadolinium with reports showing gadolinium deposits in the brain after repeated contrast-enhanced MRI examinations [14, 15]. The potential morbidity, if any, of these deposits has not yet been established but is currently being investigated. With respect to $\mathrm{x}$-ray contrast agents, it is well known that iodinated contrast agents can lead to adverse events, ranging from allergic reactions to contrast-induced nephropathy [16]. Microbubbles, on the other hand, constitute a contrast agent with a high safety profile that is used in ultrasound imaging, especially applied to cardiac, liver, and breast imaging [17, 18]. The bubbles create contrast by reflecting the ultrasound waves but also inhere the possibility to scatter $\mathrm{x}$-rays and could therefore potentially be used as a contrast agent in GIM. Accordingly, microbubbles have been shown to give detectable contrast using phase-contrast imaging with synchrotron-radiation imaging [19-21] and with a preclinical grating-interferometry $x$-ray system [22], but have not previously been shown using a clinically compatible grating-interferometry $\mathrm{x}$-ray system.

The purpose of this ex vivo study was to explore if microbubbles could be used as a contrast agent in clinically compatible GIM.

\section{Methods}

In this exploratory proof-of-mechanism study, we used $e x$ vivo phantoms consisting of four fresh chicken breasts $(5 \times 5 \times 3 \mathrm{~cm})$. The chicken tissue is dense and could therefore be considered to represent a dense breast.
Fragments of eggshells of approximately $0.5-1 \mathrm{~mm}$ were placed on top of the chicken simulating a calcification cluster and to be used as a positive contrast reference. Two different commercially available contrast agents were employed: a microbubble ultrasound contrast agent (SonoVue $8 \mu \mathrm{l} / \mathrm{ml}$, Bracco, Milan, Italy) and an iodinated contrast agent (Iopamiro $370 \mathrm{mg} / \mathrm{ml}$, Bracco, Milan, Italy). The mean diameter of SonoVue's microbubbles is $2.5 \mu \mathrm{m}$, and more than $90 \%$ of the bubbles are smaller than $8 \mu \mathrm{m}$ [23]. Two millilitres of undiluted microbubbles and iodine were respectively injected directly into the phantoms at two different locations. Native and post-contrast images were obtained.

\section{Image acquisition}

Images were acquired using a clinically compatible GIM prototype $[2,24]$. The system was based on a Philips MicroDose Mammography system (Philips Health Systems, Kista, Sweden) that had been modified with the insertion of a grating interferometer (Fig. 1). The attenuation and DPC and DF signals were extracted from the measured interference pattern, thanks to a slit-scanning phase retrieval algorithm [24]. These signals were reconstructed through an iterative method [24] and postprocessed with band removal and median-filtering to remove noise. The system was operated at $38 \mathrm{kVp}$ with an acquisition time of $14 \mathrm{~s}$. The total examination time, including handling of the sample, was estimated to roughly $7 \mathrm{~min}$. The air Kerma, measured with a RaySafe X2 device (Unfors RaySafe AB, Billdal, Sweden), was $4 \mathrm{mGy}$, which translates into a mean glandular dose of $1.3 \mathrm{mGy}$, taking into account the phantom thickness and assuming a glandular density of $100 \%$ [25].

\section{Contrast assessment}

The visibility of the contrast agent in the attenuation and DF mode was analysed in a side-by-side comparison by three experienced ( $>5$ years) breast radiologists in consensus. In addition, the contrast-to-noise-ratio (CNR) for microbubbles and iodine was calculated. Region-of-interests (ROIs) were placed adjacent to the needle tip to include the visible distribution of the bubbles, and in the centre of the iodine distribution. The following formula was used to calculate the CNR for each pixel of the contrast agent ROI:

$$
\mathrm{CNR}_{\text {pixel }}=\left(\frac{\left|I_{\mathrm{CA}}-\mu_{\text {tissue }}\right|}{\sigma_{\text {tissue }}}\right)
$$

where $I_{\mathrm{CA}}$ corresponds to the pixel-wise intensity of the contrast agent ROI, while $\mu_{\text {tissue }}$ and $\sigma_{\text {tissue }}$ are the mean and standard deviation of the tissue ROI, respectively. Afterwards, the mean and standard deviation of $\mathrm{CNR}_{\text {pixel }}$ were calculated. 


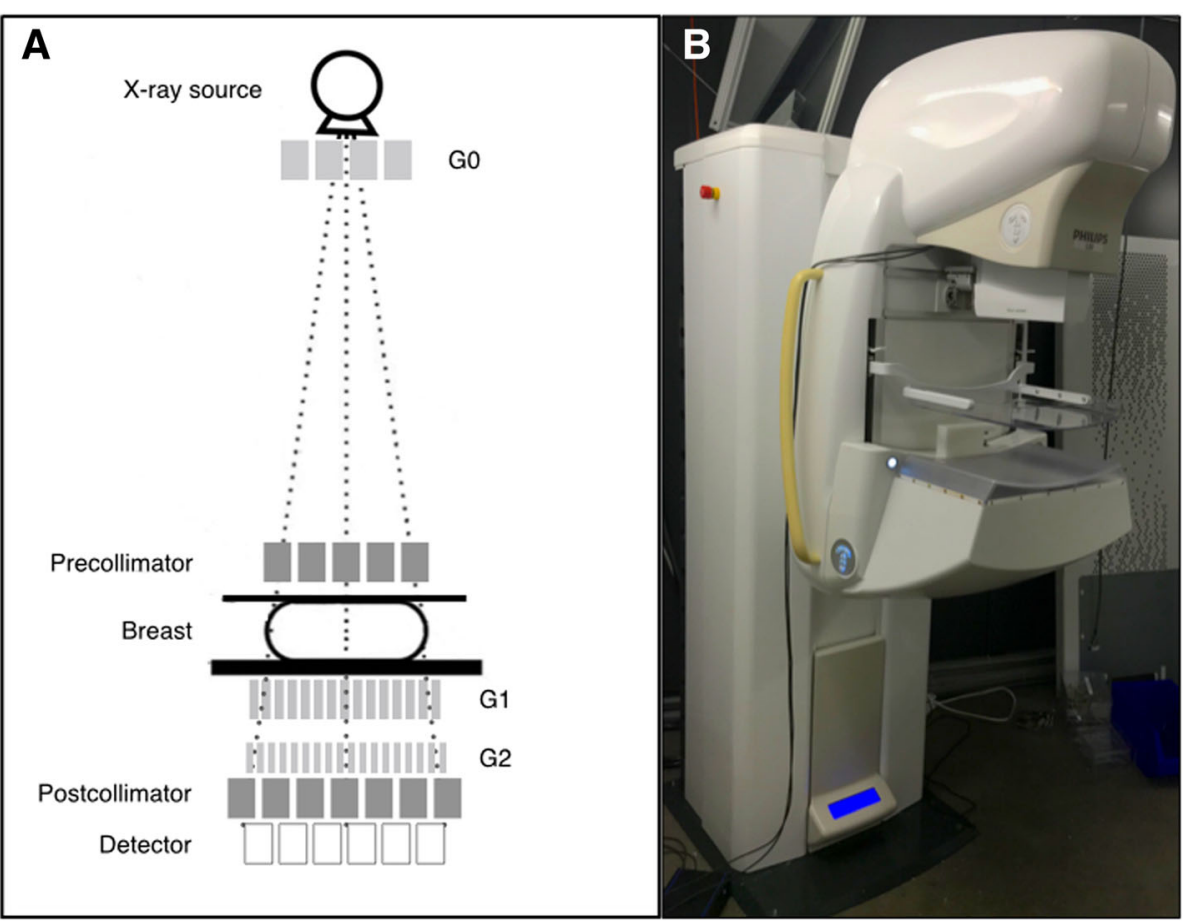

Fig. 1 a Sketch of the grating interferometer installed in a Philips MicroDose mammography system. The gratings are thin diffraction elements placed in the beam perpendicular to the optical axis, containing periodical line structures that absorb x-rays and/or shift their phase. A source grating (G0), a beam splitter grating (G1), and an analyser grating (G2) contribute at different steps to the recording of the phase and dark-field signals. b Photo of the grating interferometry mammography investigational device used in the study

\section{Results}

The radiologists judged the microbubbles to be visible in the DF image in all four samples. The microbubbles were not visible in the attenuation image. The iodine was visible both in the attenuation and DF images, but with a much higher contrast in the former (Fig. 2a). The mean CNR was $3.1 \pm 1.9$ for microbubbles in the DF mode and $24.2 \pm 6.5$ for iodine in attenuation mode (Table 1). Even if the microbubbles had a lower CNR in the DF image compared to the iodine in the attenuation mode, the background in the DF image was homogeneous making the microbubbles stand out (Fig. 2b).

\section{Discussion}

In this exploratory, proof-of-mechanism, ex vivo study, we have shown that a microbubble contrast agent can give contrast enhancement in clinically compatible GIM. Both the iodine and the microbubbles were visible in GIM, but detectable with two different image signals. This illustrates one of the key features of the GIM technique, namely, that it provides a set of three different images, each based on different physical properties, hence, potentially carrying different information: iodine is visible in the attenuation image because of its high attenuation coefficient while microbubbles become visible in the DF image due to its generation of ultra-small-angle scattering.
Since microbubbles do not attenuate $\mathrm{x}$-rays, the attenuation image can be used for anatomical orientation and the corresponding DF image for the assessment of contrast enhancement, which would result in a contrast-enhanced mammogram at a lower radiation dose and with a shorter acquisition time compared to conventional contrast-enhanced mammography [26]. Furthermore, microbubbles have different contrast kinetics compared to gadolinium and iodine than can be exploited in GIM. The assessment of wash-in and wash-out curves in the differentiation between benign and malignant lesions is a valuable tool in breast MRI, but the same has not been proven for iodine contrast-enhanced mammography [27]. As opposed to an early gadolinium washout signifying malignancy, microbubbles have been shown to have a persistent enhancement in malignant lesions [28], making it suitable for a bilateral mammography procedure. Finally, since microbubbles can be loaded with, e.g. drugs or genes, in combination with unloaded bubbles for imaging, there is a potential use also in theranostics $[29,30]$.

We found that the mean CNRs of the microbubbles were lower to that of iodine, which was expected especially considering that both contrast agents were used in its undiluted form. Iodine is normally diluted in clinical practice, whereas microbubbles are not. The main aim of this 


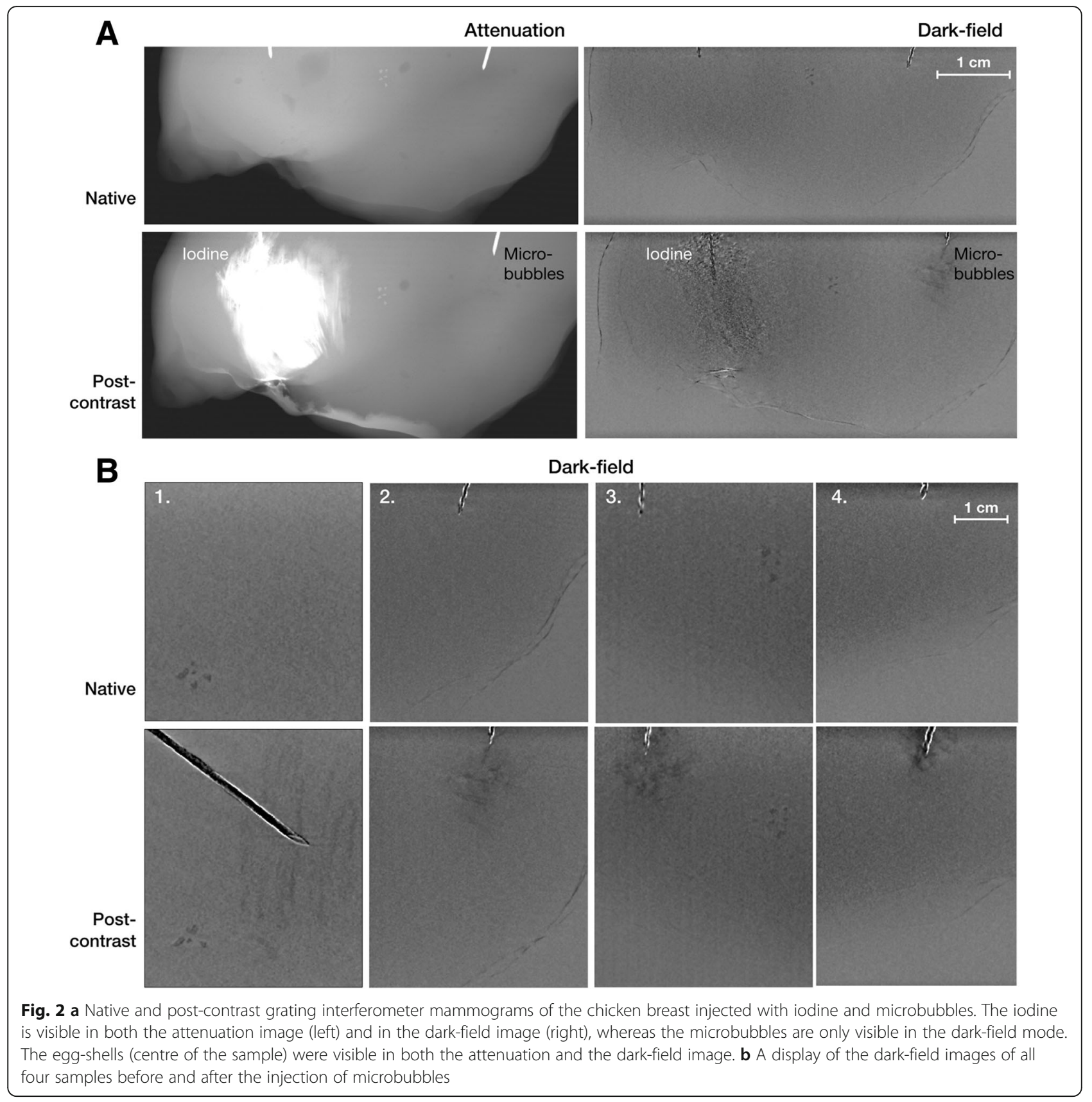

Table 1 Contrast-to-noise-ratio of the contrast agents calculated in both the attenuation and dark-field modes

\begin{tabular}{|c|c|c|c|c|}
\hline \multirow{2}{*}{$\begin{array}{l}\text { Sample } \\
\text { number }\end{array}$} & \multicolumn{2}{|l|}{ Attenuation } & \multicolumn{2}{|l|}{ Dark-field } \\
\hline & lodine & Microbubbles & lodine & Microbubbles \\
\hline 1 & $9.7 \pm 2.1$ & $1.4 \pm 0.3$ & $3.8 \pm 2.4$ & $2.7 \pm 1.3$ \\
\hline 2 & $46.0 \pm 11.7$ & $1.7 \pm 0.7$ & $5.1 \pm 2.8$ & $2.9 \pm 1.6$ \\
\hline 3 & $25.8 \pm 8.7$ & $1.8 \pm 0.9$ & $1.9 \pm 1.6$ & $1.7 \pm 1.3$ \\
\hline 4 & $15.1 \pm 3.3$ & $2.7 \pm 0.4$ & $2.8 \pm 2.2$ & $5.1 \pm 3.5$ \\
\hline Mean & $24.2 \pm 6.5$ & $1.9 \pm 0.6$ & $3.4 \pm 2.3$ & $3.1 \pm 1.9$ \\
\hline
\end{tabular}

Data are given as mean \pm standard deviation study was, however, only to determine whether there was a visible enhancement with the microbubbles in DF mode or not. Concerning test reliability, the experiment was repeated four times, and in all cases, the microbubbles were visible in the dark-field mode. However, there were several factors that led to a variation in the measurements such as the force applied to the contrast injection and a variation in sample thickness. Most importantly, the samples were not homogeneous leading to an uneven infiltration of the directly injected contrast agents.

If microbubbles are to be used as a contrast agent in GIM in vivo, further considerations have to be made 
concerning dose, and most importantly, the proper size of the bubbles. Theoretically, since the DF signal in grating interferometry is usually generated from small- or ultra-small-angle scattering, bubbles whose diameter matches the auto-correlation length of the setup could optimise the scattering signal $[31,32]$. Consequently, the optimal bubble size for our investigational device would be $1 \mu \mathrm{m}$, i.e. slightly smaller than the commercial contrast agent actually used in the experiment.

To the best of our knowledge, this is the first time the feasibility of using microbubbles in a clinically compatible grating-interferometry phase-contrast imaging set-up has been shown. Velroy et al. [22] found that microbubbles scatter $\mathrm{x}$-rays using grating-interferometry phase-contrast imaging in an experimental setting imaging vials with microbubbles with acquisition time and dose not optimised for clinical imaging. It is expected that the CNR of the DF contrast can be higher with a preclinical system compared with a clinical system, since the latter has constraints on geometry, radiation dose, and acquisition time. Without these constraints, the reduction of photon flux caused by the gratings can be compensated in the pre-clinical set-up to reduce the noise, by increasing the exposure time or making the interferometer shorter; in addition, since it is usually not subject to very strict geometric constraints, there is more freedom to optimise the geometry in terms of grating periods and inter-grating distances in order to have the most appropriate auto-correlation length.

There have been several studies on the DF signal as a function of the particle size [33-36]. For instance, Gkoumas et al. [36] measured colloidal suspensions of $\mathrm{SiO}_{2}$ microspheres of two different diameters $(1.86 \mu \mathrm{m}$ and $7.75 \mu \mathrm{m})$ in glycerine with increasingly higher concentrations $(5-40 \%)$ on a synchrotron grating interferometer with an autocorrelation length of about $3.0 \mu \mathrm{m}$. They found that the utilised grating interferometer setup was more sensitive to higher concentrations. This outcome must be validated for our case, taking into account that we are measuring microbubble solution meant to be intravenously injected and that we are using a polychromatic setup.

However, even if we have demonstrated that microbubbles can provide visible contrast enhancement with a clinically compatible GIM device, the question whether this is a viable alternative in vivo remains to be answered and constitutes the main limitation of our study at this point. Additional studies on the appropriate bubble size as well as contrast agent concentration for GIM are needed.

In conclusion, GIM is a novel breast imaging technique at the edge of being clinically implemented. The method generates a set of three images based on the attenuation, refraction, and scattering of $\mathrm{x}$-rays, which opens up the use of various contrast agents. This experiment set out to investigate whether microbubbles gave contrast enhancement ex vivo using a clinically compatible GIM setup. We found that microbubbles, due to their scattering properties, gave contrast enhancement in the dark-field mode. This implies the potential use of a contrast agent with a high safety profile, and with the prospect of using theranostics, in $x$-ray-based breast imaging.

\section{Abbreviations}

CNR: Contrast-to-noise-ratio; DF: Dark-field; DPC: Differential phase contrast; GIM: Grating interferometry mammography; MRI: Magnetic resonance imaging; ROl: Region of interest

\section{Funding}

This project has been supported by the ERC grant ERC-2012-StG 310005 "PhaseX", the SNF-Sinergia CRS112-154472 "MedXPhase", the SNF-Sinergia CRSII5_183568 "GI-BCT" grant, SNF R'Equip grant 206021_177036, and the Swedish Society of Medical Research.

\section{Availability of data and materials}

The dataset used and/or analysed during the current study is available from the corresponding author on reasonable request.

\section{Authors' contributions}

RK and MS conceived the idea of the study. KL, CA, RK, and SF planned the study. KL, CA, and SF performed the experiment. ZW, MS, SP, TK NK, BD, and $\mathrm{KJ}$ participated in the technical implementation. $\mathrm{KL}$ and $\mathrm{CA}$ analysed the data and wrote the manuscript. All authors contributed to the revision of the manuscript. All authors read and approved the final manuscript.

Ethics approval and consent to participate

Not applicable.

Consent for publication

Not applicable.

Competing interests

$\mathrm{SP}, \mathrm{TK}, \mathrm{NK}$, and BD are employees of Philips GmbH Innovative Technologies. All other authors declare that they have no competing interests.

\section{Publisher's Note}

Springer Nature remains neutral with regard to jurisdictional claims in published maps and institutional affiliations.

\section{Author details}

${ }^{1}$ Swiss Light Source, ETH Zurich, Paul Scherrer Institute, 5232 Villigen, Switzerland. ${ }^{2}$ Institute for Biomedical Engineering, University and ETH Zurich, Zurich, Switzerland. ${ }^{3}$ Department of Radiology, Kantonsspital Baden, Im Ergel

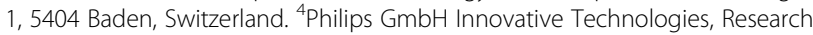
Laboratories, Philips Research Hamburg, Röntgenstrasse 24-26, 22335 Hamburg, Germany.

Received: 30 October 2018 Accepted: 4 April 2019

Published online: 21 May 2019

\section{References}

1. Bravin A, Coan P, Suortti P (2013) X-ray phase-contrast imaging: from preclinical applications towards clinics. Phys Med Biol 58:R1-35. https://doi.org/ 10.1088/0031-9155/58/1/r1

2. Arboleda C, Wang Z, Koehler T et al (2017) Sensitivity-based optimization for the design of a grating interferometer for clinical $\mathrm{X}$-ray phase contrast mammography. Opt Express 25:6349-6364. https://doi. org/10.1364/oe.25.006349

3. Weitkamp T, Diaz A, David C et al (2005) X-ray phase imaging with a grating interferometer. Opt Express 13:6296-6304. https://doi.org/10.1364/ OPEX.13.006296

4. Keyriläinen J, Bravin $A$, Fernández $M$, Tenhunen $M$, Virkkunen $P$, Suortti $P$ (2010) Phase-contrast X-ray imaging of breast. Acta Radiol 51:866-884 https://doi.org/10.3109/02841851.2010.504742 
5. Stampanoni M, Wang $Z$, Thüring $T$ et al (2011) The first analysis and clinical evaluation of native breast tissue using differential phase-contrast mammography. Invest Radiol 46:801-806. https://doi.org/10.1097/RLI. Ob013e31822a585f

6. Grandl S, Scherer K, Sztrókay-Gaul A et al (2015) Improved visualization of breast cancer features in multifocal carcinoma using phase-contrast and dark-field mammography: an ex vivo study. Eur Radiol 25:3659-3668. https://doi.org/10.1007/s00330-015-3773-5

7. Scherer K, Willer K, Gromann L et al (2015) Toward clinically compatible phase-contrast mammography. PLoS One 10:e0130776. https://doi.org/10. 1371/journal.pone.0130776

8. Hauser N, Wang Z, Kubik-Huch RA et al (2014) A study on mastectomy samples to evaluate breast imaging quality and potential clinical relevance of differential phase contrast mammography. Invest Radiol 49:131-137. https://doi.org/10.1097/rli.0000000000000001

9. Wang Z, Hauser N, Singer G et al (2014) Non-invasive classification of microcalcifications with phase-contrast X-ray mammography. Nat Commun 5:3797. https://doi.org/10.1038/ncomms4797

10. Scherer K, Braig E, Ehn S et al (2016) Improved diagnostics by assessing the micromorphology of breast calcifications via X-ray dark-field radiography. Sci Rep 6:36991. https://doi.org/10.1038/srep36991

11. Wang Z, Hauser N, Singer G et al (2016) Correspondence: reply to 'Quantitative evaluation of X-ray dark-field images for microcalcification analysis in mammography. Nat Commun 7:10868. https://doi.org/10.1038/ ncomms 10868

12. Tagliafico AS, Bignotti B, Rossi F et al (2016) Diagnostic performance of contrast-enhanced spectral mammography: systematic review and metaanalysis. Breast 28:13-19. https://doi.org/10.1016/j.breast.2016.04.008

13. Christiansen C (2005) X-ray contrast media--an overview. Toxicology 209: 185-187. https://doi.org/10.1016/j.tox.2004.12.020

14. Kanda T, Nakai Y, Oba H, Toyoda K, Kitajima K, Furui S (2016) Gadolinium deposition in the brain. Magn Reson Imaging 34:1346-1350. https://doi.org/ 10.1016/j.mri.2016.08.024

15. Berger F, Kubik-Huch RA, Niemann T (2018) et al Gadolinium distribution in cerebrospinal fluid after administration of a gadoliniumbased MR contrast agent in humans. Radiology 288:703-709. https://doi. org/10.1148/radiol.2018171829

16. Morcos SK, Thomsen HS (2001) Adverse reactions to iodinated contrast media. Eur Radiol 11:1267-1275. https://doi.org/10.1007/s003300000729

17. Wei K, Mulvagh SL, Carson L et al (2008) The safety of deFinity and Optison for ultrasound image enhancement: a retrospective analysis of 78,383 administered contrast doses. J Am Soc Echocardiogr 21:1202-1206. https:// doi.org/10.1016/j.echo.2008.07.019

18. Appis AW, Tracy MJ, Feinstein SB (2015) Update on the safety and efficacy of commercial ultrasound contrast agents in cardiac applications. Echo Res Pract 2:R55-R62. https://doi.org/10.1530/erp-15-0018

19. Tang R, Xi Y, Chai WM et al (2011) Microbubble-based synchrotron radiation phase contrast imaging: basic study and angiography applications. Phys Med Biol 56:3503-3512. https://doi.org/10.1088/0031-9155/56/12/004

20. Wu D, Wong MD, Yang K et al (2017) Using microbubble as contrast agent for high-energy $\mathrm{X}$-ray in-line phase contrast imaging: demonstration and comparison study. IEEE Trans Biomed Eng. https://doi.org/10.1109/tbme. 2017.2741942

21. Arfelli F, Rigon L, Menk RH (2010) Microbubbles as x-ray scattering contrast agents using analyzer-based imaging. Phys Med Biol 55:1643-1658. https:// doi.org/10.1088/0031-9155/55/6/008

22. Velroyen A, Bech M, Tapfer A et al (2015) Ex vivo perfusion-simulation measurements of microbubbles as a scattering contrast agent for gratingbased X-ray dark-field imaging. PLoS One 10:e0129512. https://doi.org/10. 1371/journal.pone.0129512

23. Schneider M (1999) Characteristics of SonoVue ${ }^{\mathrm{TM}}$. Echocardiography 16:743746. https://doi.org/10.1111/j.1540-8175.1999.tb00144.x

24. Thomas K, Heiner D, Gerhard M et al (2015) Slit-scanning differential x-ray phase-contrast mammography: proof-of-concept experimental studies. Med Phys 42:1959-1965. https://doi.org/10.1118/1.4914420

25. Boone JM (1999) Glandular breast dose for monoenergetic and high-energy X-ray beams: Monte Carlo assessment. Radiology 213:23-37. https://doi.org/ 10.1148/radiology.213.1.r99oc3923

26. Jeukens CR, Lalji UC, Meijer E et al (2014) Radiation exposure of contrastenhanced spectral mammography compared with full-field digital mammography. Invest Radiol 49:659-665. https://doi.org/10.1097/rli. 0000000000000068

27. Diekmann F, Diekmann S, Jeunehomme F, Muller S, Hamm B, Bick U (2005) Digital mammography using iodine-based contrast media: initial clinical experience with dynamic contrast medium enhancement. Invest Radiol 40: 397-404. https://doi.org/10.1097/01.rli.0000167421.83203.4e

28. Zhao H, Xu R, Ouyang Q, Chen L, Dong B, Huihua Y (2010) Contrastenhanced ultrasound is helpful in the differentiation of malignant and benign breast lesions. Eur J Radiol 73:288-293. https://doi.org/10.1016/j. ejrad.2009.05.043

29. Paefgen $V$, Doleschel D, Kiessling F (2015) Evolution of contrast agents for ultrasound imaging and ultrasound-mediated drug delivery. Front Pharmacol 6:197. https://doi.org/10.3389/fphar.2015.00197

30. Millard TP, Endrizzi M, Rigon L et al (2013) Quantification of microbubble concentration through $\mathrm{x}$-ray phase contrast imaging. Appl Phys Lett 103: 114105. https://doi.org/10.1063/1.4821277

31. Malecki A, Potdevin G, Pfeiffer F (2012) Quantitative wave-optical numerical analysis of the dark-field signal in grating-based X-ray interferometry. EPL (Europhysics Letters) 99:48001. https://doi.org/10.1209/0295-5075/99/48001

32. Kagias M, Wang Z, Jefimovs K, Stampanoni M (2017) Dual phase grating interferometer for tunable dark-field sensitivity. Appl Phys Lett 110:014105. https://doi.org/10.1063/1.4973520

33. Yashiro W, Terui Y, Kawabata K, Momose A (2010) On the origin of visibility contrast in X-ray Talbot interferometry. Opt Express 18:16890-16901. https:// doi.org/10.1364/oe.18.016890

34. Lynch SK, Pai V, Auxier J et al (2011) Interpretation of dark-field contrast and particle-size selectivity in grating interferometers. Appl Opt 50:4310-4319. https://doi.org/10.1364/ao.50.004310

35. Strobl M (2014) General solution for quantitative dark-field contrast imaging with grating interferometers. Sci Rep 4:7243. https://doi.org/10.1038/ srep07243

36. Gkoumas S, Villanueva-Perez P, Wang Z, Romano L, Abis M, Stampanoni M (2016) A generalized quantitative interpretation of dark-field contrast for highly concentrated microsphere suspensions. Sci Rep 6:35259. https://doi. org/10.1038/srep35259

\section{Submit your manuscript to a SpringerOpen ${ }^{\circ}$ journal and benefit from:}

- Convenient online submission

- Rigorous peer review

- Open access: articles freely available online

High visibility within the field

- Retaining the copyright to your article

Submit your next manuscript at $>$ springeropen.com 